\title{
CSAIL
}

Computer Science and Artificial Intelligence Laboratory

Massachusetts Institute of Technology

\section{Developing and Refining an Adaptive Token-Passing Strategy}

Burkhard Englert, Larry Rudolph, Alex Shvartsman

2001, June

\section{Computation Structures Group \\ Memo 440}

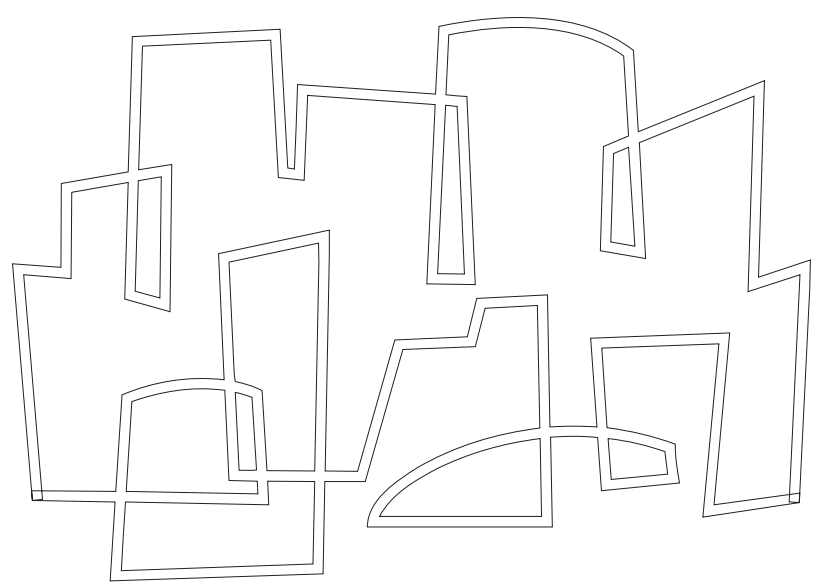

The Stata Center, 32 Vassar Street, Cambridge, Massachusetts 02139 



\title{
Developing and Refining an Adaptive Token-Passing Strategy
}

\author{
Burkhard Englert * $\quad$ Larry Rudolph ${ }^{\dagger} \quad$ Alex Shvartsman ${ }^{\ddagger}$
}

\begin{abstract}
Token rotation algorithms play an important role in distributed computing, to support such activities as mutual exclusion, round-robin scheduling, group membership and group communication protocols. Ring-based protocols maximize throughput in busy systems, but can incur a linear, in the number of processors, delay when a processor needs to obtain a token to perform an operation.

This paper synthesizes these two algorithmic techniques thereby improving performance (responsiveness) of logical ring protocols. The parameterized technique preserves the safety properties of ring protocols and maintains high throughput in busy systems, while reducing the delay in lightly loaded systems from a linear to the logarithmic function in the number of processors. The algorithmic development is done using term rewriting systems where our parameterized protocol is developed in a series of safetypreserving refinements of a basic specification.
\end{abstract}

\section{Introduction}

Developing adaptive, token-based protocols for mutual exclusion, group communication, and other distributed algorithms, is difficult because of the subtle interplay of correctness and performance concerns. We develop a generic token-based protocol that can give rise to many implementations, each optimized for a unique set of performance characteristics but all having the same safety criteria. The protocols are developed from a high-level description in which the desired properties are basically self evident. A succession of refinements, each of which is easily shown to be as safe as the previous, are presented. Separating correctness from performance, makes it easier to devise complex sets of rules to improve performance without constantly worrying about how they impact correctness.

The final refined protocol results in a most responsive system and is described in just 8 rules. It is a token-based

* University of California Los Angeles, Dept. of Mathematics, Los Angeles, CA 90095-1555. Email: englert@math. ucla.edu.

† Massachusetts Institute of Technology, Laboratory for Computer Science, 545 Technology Square, Cambridge, MA 02139. Email: rudolphelcs.mit.edu. The work of this author was in part supported by DARPA under the Air Force Research Lab contract F30602-992-0511.

$\ddagger$ University of Connecticut, Computer Science and Eng., 191 Auditorium Road, U-155, Storrs, CT 06269. Email: a as @ cse.uconn. edu. The work of this author was in part supported by a NSF CAREER Award 9984778, NSF Grant 9988304 and a grant from AFOSR. algorithm that combines passing a token around a ring of processors with a logarithmic, binary search strategy. Like the IEEE 802.4 protocol, ring-based schemes provide good throughput under varied loads, stable performance under increasing loads, and ensures fair access with deterministic guarantees. On the other hand, logarithmic, tree-based protocols, provide fair access on average for moderate loads and excellent response when the use is bursty but infrequent. Our adaptive scheme provides the best of both providing good and fair performance for loaded systems and quick, logarithmic response under light to moderate loads. A token flows around the ring, as in the usual ring based approach, however, when some node wants the token, search messages and traps are used so that when the token is found, it can be quickly forwarded to a needy node.

This paper can be seen as an example of a technique for structuring distributed application systems as abstract components that may be mapped to realistic distributed architectures. The components are designed to implement specific application properties. It is desireable for the properties of the constituent components to be "orthogonal" in the sense that the analysis of the resulting system can be derived from the analysis of the individual components.

Given application requirements, our system can make use of two qualitatively different communication modes:

(1) Components use "expensive" communication services; the object here is to use the guarantees of these services to show the correctness/safety properties.

(2) Components use "cheap" messaging without firm guarantees to shepherd the overall system so as to enhance its performance, e.g., if the system maintains a circulating token, the cheap messages may be used as hints designed to alter the token rotation schedule to divert the token to the nodes with greater needs.

The correctness of the system is shown by considering only the "expensive" messages, while the cheap messages do not affect correctness. In particular, the system remains correct even if no "cheap" message is ever sent. The "cheap" messages are used to show conditional performance property of the system. In general we argue if a certain number of "cheap" messages are sent and are delivered within certain time bounds, then the system can achieve desired performance goals, e.g., the overall distributed computation makes progress or that it makes progress at a known pace.

We consider a set of fully interconnected nodes where, at any time, a node may wish to obtain an exclusive possession 
of a broadcast medium in order to multicast to all nodes, or to acquire exclusive access to some shared resource, in the same global order. For expository purposes, it is simplier to think in terms of broadcasting even though all our results are applicable to mutual exclusion.

Our token-passing solution incorporates an interesting duality (push-pull). When a node requires the token, it can either actively try to find the token or the owner of a token can actively try to find which node requires it. In either case, combining a rotation strategy with a binary search reduces the number of messages. It achieves a responsiveness, defined as the maximum time between some service request and the satisfaction of some (possibly different) request, of $O(\log N)$ under all loads and is fair.

Our protocols are described in a purely syntactic fashion making use of a Term Rewriting System. The proofs of safety are also purely syntactic. Associating a meaning to the syntax, gives rise to a family of implementations.

\subsection{Related work}

Mutual exclusion protocols [10, 16, 18] are very important in enabling cooperative distributed computation. Tokenbased approaches $[3,5,7,8,9,13]$ have been used in designing mutual exclusion and resource management protocols for a long time. These token-based approaches include ring-oriented solutions, which require up to a linear number of messages, and tree-oriented solutions that require the number of messages linear in the diameter of the graph.

An important example of cooperative distributed systems is provided by group communication services (GCS) [6]. GCSs enable processes of a distributed system to operate collectively as a group. Different GCSs offer different guarantees about the order and reliability of message delivery, see the survey [19]. Logical token ring approaches to establish message ordering and to maintain membership have been used in GCSs, e.g., [1].

Another related problem is called mutual search [4]. In this problem, a set of agents distributed in a network need to locate each other by querying the nodes of the network. Here the model can be interpreted as a set of clients (token requests) searching for resources (tokens). (See also the related problems given in the appendix in [4].)

\subsection{Paper Organization}

The structure of the rest of this paper is as follows. The formal foundations, definitions, and notation are first presented in Section 2. Section 3 presents the basic protocol and its refinements leading to a local history protocol using token passing. Performance issues are addressed in Section 4. Section 5 concludes with a discussion and extensions.

\section{Foundations}

Our protocols are described informally as well as in a precise symbolic manner. This section describes our model of computation and the formal protocol specification notation.

The distributed computing setting is assumed to consist of a finite set of processors communicating by means of message passing and sharing no common storage. The processors have unique identifiers from a finite set $\mathcal{P}$, where $N=|\mathcal{P}|$ is the number of processors, and the communication network is a complete graph. We assume complete asynchrony and make no assumptions about time or communication delays when we reason about the safety properties. However, to establish the performance results we involve operational reasoning about executions assuming that the communication occurs within bounded delays and that local events take negligible time.

We use Term Rewriting Systems (TRS's) to define our protocol. TRS's have been successfully used to model microarchitectures [2] and to specify cache coherence protocols [17]. A TRS $\mathcal{T}=(\Sigma, R)$ consists of a set of terms $\Sigma$ and a set of rewriting rules $\mathcal{R}$. The terms represent system states and the rules specify state transitions. The general structure of rewriting rules is as follows:

$$
s_{1} \rightarrow s_{2} \quad\left(\text { if } p\left(s_{1}\right)\right)
$$

where $s_{1}$ and $s_{2}$ are terms and $p\left(s_{1}\right)$ is an optional predicate about $s_{1}$.

A rule can be used to rewrite a term if its left-hand-side pattern matches the term or one of its subterms, and the corresponding predicate, if any, is true. If several rules are applicable, then any one of them may be applied. If no rule is applicable, then the term cannot be rewritten any further. A rewriting strategy can be used to specify which rule among the applicable rules should be applied at each rewriting step. A sequence of terms (possibly infinite), starting with some initial term and obtained by successive application of rules is called a reduction. A path is a subsequence of a reduction.

Notation: It is important to distinguish between variables and constants while pattern matching. A variable matches any expression while a constant matches only itself. We will follow the convention where variables and constants are represented by identifiers that consist of English and Greek letter, respectively. Identifiers with upper case letters are variables, and usually represent sets. We use '-', the wild-card term, as a placeholder for any possible term. When a wild-card appears in the same position on both sides of the re-rewrite rule, then we assume that it is left unchanged by the rule (this convention serves solely to unclutter the rules by reducing the number of symbols). We use 'I' as a term catenation connective where the ordering does not matter (i.e., '|' is associative and commutative). Additional notation used throughout this paper is given in Figure 1.

Our systems will be described from a global point of view. Hence to ensure safety we need to ensure that all local events at a node are captured by our system description, our set of rules. We formalize this with the following definitions:

Definition $1 A$ history is a sequence of events of interest that are observed locally. A node may also receive a history in a message from another node, and it can append a local event to the history. 
- The set of processors is $\mathcal{P}$.

- Given a cycle graph $(\mathcal{P}, \mathcal{E})$ and $x \in \mathcal{P}$, we define $x^{+1}$ to be $y$ such that $(x, y) \in \mathcal{E}$. In the same vein, $x^{+2}$ is $\left(x^{+1}\right)^{+1}$, $x^{+n}$ is the $n^{t h}$ successor of $x$, and $x^{-n}$ is such $y$ that $y^{+n}=x$.

- The notation $\|_{p(j)} x(j)$ denotes an unordered list of terms $x(j)$ such that for each $j$ the predicate $p(j)$ is satisfied. E.g., $\|_{j \in\{1,2,3\}} j$ stands for $1|2| 3$.

- $\oplus$ is the append operator.

- We define $\phi_{x}$ to be the distinguished symbol for each $x \in \mathcal{P}$. It identifies $x$ and it serves as the left identity for $\oplus$; we use to denote an empty token request for $x$.

- We define $\tau_{x}$ to be the distinguished symbol for each $x \in \mathcal{P}$. We use $\tau_{x}$ to denote a token trap set on behalf of processor $x$.

- Let $A$ and $B$ be (ordered) sequences over $\mathcal{C} \cup \mathcal{D}$. We define the relation ' $\subset$ ' so that $A \subset B$ when $A$ is a prefix sequence of $B$, and we define the relation ' $\subset_{\mathcal{C}}$ ' such that $A \subset_{\mathcal{C}} B$ when $A$ is a prefix sequence of $B$ once they are projected onto the elements of $\mathcal{C}$.

Figure 1: Definitions and brief explanation of additional notation.

Definition 2 A protocol is said to satisfy the prefix property if each node's individual history is a prefix of the global history.

\section{Basic Protocols}

The high level global specification is presented. It is then refined in three steps to eliminate any global state or instantaneous actions. The refinement steps are small making it easy to prove that they maintain the safety property.

\subsection{Global Specification - System S}

At any time, a node can decide it needs to broadcast and becomes a ready node. The data of some ready node is broadcast to all the nodes. Two rules capture these two basic operations and define our first protocol, System S. We consider the set $Q$ to be the set of nodes and their broadcast data, encoded as pairs $\left(x, d_{x}\right)$. The intention is that $x$ is a node and $d_{x}$ is the data it wishes to broadcast. Initially, each node has no data to broadcast, and so $Q$ is initialized to $N$ pairs $\left(x, \phi_{x}\right)$, where $\phi_{x}$ is a distinguished no-message symbol for each node. The first rule captures the notion of a node wanting to broadcast some data - the approriate pair in $Q$ is updated to contain the new data to broadcast.

Data is broadcast to all the nodes by appending it to the history $\log$ of all broadcast messages. This is captured by rule 2, that removes some pair from $Q$ and appends the data to a history $\log$ of messages, $H$. Figure 2 gives a purely symbolic, precise description.

We note that rule 2 ensures that System $S$ satisfies the prefix property. From now on, we will use this fact to obtain the safety property for more refined systems.

\subsection{Local Histories - System S1}

Our first refinement is a prepatory step that will led to an elimination of the global history. We introduce local histories, $P$, maintained by each node. In System $\mathrm{S} 1$ the global history is copied to local history records. A node may perform this copy at any time - it is a performance issue as to exactly when the copy should happen, but from a safety point of view, the nodes can perform a copy in any order and at any time.

System S1 consists of three rules, see Figure 3, the first two are essentially the same as in System S. The third rule, which can be invoked at any time, copies the global history to some node's local, prefix history

Lemma 1 S1 satisfies the prefix property.

Proof sketch: Given two states in $\mathrm{S} 1, C$ and $D$, such that there is a path from $C$ to $D$, one can map each state in $\mathrm{S} 1$ to a state in $\mathrm{S}$ under a mapping $M$ and it is easy to show that there is a path from $M(C)$ to $M(D)$ in $\mathrm{S}$. Hence since $\mathrm{S}$ satisfies the prefix property, $\mathrm{S} 1$ does. The mapping is trivial, just ignore the values of $P$.

Exactly how and when a node updates its prefix history can be established either with additional rules or by refining the current rules. Although this will limit the number of possible states that the system can enter, it will not violate the correctness properties.

\subsection{Token Passing - System Token}

The broadcast rule can be restricted to the time when a node has the token. The transitions and reachable states of such a system, like the one represented as System Token in Figure 4, are clearly a proper subset of the transitions and 
System S

\begin{tabular}{|r|lllll|}
\hline 0 & Initial state $(Q, H)$ & $:$ & $\left(\|_{x \in \mathcal{P}}\left(x, \phi_{x}\right)\right.$, & $\varnothing)$ \\
\hline 1 & $\left(Q \mid\left(x, d_{x}\right)\right.$, & $-)$ & $\rightarrow$ & $\left(Q \mid\left(x, d_{x} \oplus n e w_{x}\right)\right.$, & $-)$ \\
\hline 2 & $\left(Q \mid\left(x, d_{x}\right)\right.$, & $H)$ & $\rightarrow$ & $(Q$, & $\left.H \oplus d_{x}\right)$ \\
\hline
\end{tabular}

Figure 2: System S, The base, abstract protocol. When a node wishes to broadcast, it adds a new datum to $Q . H$ is the global history of the broadcasts. It is an ordered set.

System S1

\begin{tabular}{|c|c|c|c|c|c|c|c|}
\hline$\overline{0}$ & Initial stat & $\overline{e(Q)}$ & & : & $\left(\|_{x \in \mathcal{P}}\left(x, \phi_{x}\right)\right.$ & $\varnothing$ & $x \in \mathcal{P}(x, \varnothing))$ \\
\hline 1 & $\left(Q \mid\left(x, d_{x}\right)\right.$ & ,- & $-)$ & $\rightarrow$ & $\left(Q \mid\left(x, d_{x} \oplus n e w_{x}\right)\right.$ & - & $-)$ \\
\hline 2 & $(Q)\left(x, d_{x}\right)$ &,$H$, & $-)$ & $\rightarrow$ & $Q$ &,$H \oplus d_{x}$ & $-)$ \\
\hline$\overline{\overline{3}}$ & $\overline{(-}$ & $\overline{\overline{, H}}$ & $\overline{-1)(}$ & $\rightarrow$ & $\overline{(-}$ & $\overline{\bar{H}}$ & $\overline{\bar{P} \mid(y, H))}$ \\
\hline
\end{tabular}

Figure 3: System S1, The set $P$ is the collection of all the local history prefix variables. It contains pairs $\left(i, H_{i}\right)$, which can represent the local prefix history $H_{i}$ for node $i$. The first two rules are from System $\mathrm{S}$, but with an extra parameter field.

reachable states of System S1. Thus, it is easy to show that it too satisfies the prefix property.

Lemma 2 System Token satisfies the prefix property.

\subsection{A Distributed Protocol-System Message-Passing}

A somewhat less abstract protocol does not have explicit global state and uses message passing to corrolate the local states at disjoint nodes. System Message-Passing is a refinement of System Token with these two features.

The "history" global variable is not maintained in any single place, rather it is passed around the nodes as part of some message. When a message arrives at a node, the "history" component of the message node can be used by the node to update its local prefix history. In the protocol specification, we follow the convention that a set with pairs $(i, j)$ indicates that node $i$ contains data $j$, thus the set is distributed among the nodes.

We also must translate the single rule that instantaneously changes state at two different nodes, into the more realistic two rules: one for sending and another for receiving messages. Each node has some input set and an output set of messages; the totality of these sets for all nodes in the system is represented, respectively, by the input set $I$ and the output set $O$. These sets are maintained using a rule that models message passing. Once again, each of these sets consists of pairs $(i, j)$ indicating that node $i$ has messages $j$. Note that the intention is to model distributed sets of messages; the fact that these are represented by "centralized" sets $I$ and $O$ is simply due to notational convenience. The specification for System Message-Passing is given in Figure 5.

It is easy to show correctness by using the idea of drained states. A state in System Message-Passing is mapped back to a state in System Token by getting rid of states in which the transmission sets are empty, by stopping any new transmissions, and letting everything drain. We also map the maximal $H_{x}$ in System Message-Passing to $H$ in Token.

Lemma 3 System Message-Passing satisfies the prefix property.

\section{Performance Issues}

Often there are several competing criteria for optimization. Traditional protocols integrate performance with correctness making it difficult to modify a protocol to improve its performance without violating some correctness criteria. Our approach is to separate the two concerns. The basic protocol just described says nothing about performance. It assumes that the token can be passed to any node and at any time, leaving wide scope for implementation. The rules specify a large set of behaviors, some of which are highly inefficient. This section refines the set of behaviors to just those that perform well in regards to the performance criteria of waiting time and number of messages.

Let us call a node that require the token a ready node otherwise it is an idle node. It is a non-responsive system that will pass the token to many idle nodes while there are ready nodes waiting for it.

Definition 3 The Responsiveness of a system is the maximum time period during which at least one node requires the token and until the token is given to a ready node.

Note that responsiveness is not the same as average waiting time. For example, when all nodes simultaneously require the token, the responsiveness is $O(1)$, whereas the average delay can be $O(N)$ since at least half the nodes will have to wait $N / 2$ cycles before they get the token. Also note that the time period is not from the time a node becomes ready to the time the same node receives the token, but rather from the time a node becomes ready to the time 
System Token

\begin{tabular}{|c|c|c|c|c|c|c|}
\hline 0 & Initial state $(Q, H, P, T)$ & : & $\left(\|_{x \in \mathcal{P}}\left(x, \phi_{x}\right)\right.$ &,$\varnothing$ & $x \in \mathcal{P}(x, \varnothing)$ & $, x)$ \\
\hline 1 & $\left(Q \mid\left(x, d_{x}\right),-,-\right.$ & $\rightarrow$ & $\left(Q \mid\left(x, d_{x} \oplus n e w_{x}\right)\right.$ &,- & - & $,-)$ \\
\hline$\overline{2}$ & \begin{tabular}{|c|c|}
$\left.\left(Q, d_{x}\right), H, P \mid(x,-), x\right)$ \\
\end{tabular} & $\rightarrow$ & $\overline{(Q)\left(x, \phi_{x}\right)}$ & & $\overline{{ }_{,} P(x, H \oplus d}$ & , y) \\
\hline
\end{tabular}

Figure 4: System Token, The second rule is a combination of rules 2 and 3 of System S1. A token passing mechanism is represented by restricting the update to the global history record. Node $x$ can append its data only when it has the token, which is represented by the fourth (rightmost) field $T$.

System Message-Passing

\begin{tabular}{|r|ll|}
\hline 0 & Initial state $(Q, P, T, I, O)$ & $:\left(\|\mid\|_{x \in \mathcal{P}}\left(x, \phi_{x}\right), \|_{x \in \mathcal{P}}(x, \varnothing), x, \varnothing, \varnothing\right)$ \\
\hline 1 & $\left(Q \mid\left(x, d_{x}\right),-,-,-,-\right)$ & $\rightarrow\left(Q \mid\left(x, d_{x} \oplus n e w_{x}\right),-,-,-,-\right)$ \\
\hline \hline 2 & $(-,-,-, I, O \mid(x,(y, m)))$ & $\rightarrow(-,-,-, I \mid(y,(x, m)), O)$ \\
\hline \hline 3 & $\left(Q\left|\left(x, d_{x}\right), P\right|(x, H), x,-, O\right)$ & $\rightarrow\left(Q, P\left|\left(x, H \oplus d_{x}\right), \perp,-, O\right|\left(x,\left(y, H \oplus d_{x}\right)\right)\right)$ \\
\hline 4 & $(-, P|(x,-), \perp, I|(x,(y, H)),-) \rightarrow(-, P \mid(x, H), x, I,-)$ \\
\hline \hline $3^{\prime}$ & $\left(Q\left|\left(x, d_{x}\right), P\right|(x, H), x,-, O\right)$ & $\begin{array}{c}\rightarrow\left(Q, P\left|\left(x, H \oplus d_{x}\right), \perp,-, O\right|\left(x,\left(y, H \oplus d_{x}\right)\right)\right) \\
\left.\text { (where } y=x^{+1}\right)\end{array}$ \\
\hline
\end{tabular}

Figure 5: System Message-Passing, The history $H$ is no longer explicitly in the global state, but is passed around as a message. The two new parameters, $I$ and $O$, represent message passing between nodes. Each node has an input set and an output set. The output set, $O$, contains the pair $(x,(y, m))$ that represents $x$ sending a message $m$ to node $y$. The input set, $I$, is similar, where $(x,(y, m))$ means that $x$ has received a message from $y$ containing data $m$. The special distinguished symbol $\perp$ represents the case where the token is in transit and thus no node has the token. To guarantee a circular token rotation, replace rule 3 by rule $3^{\prime}$. This will be used to make certain performance guarantees.

at which a ready node, not necessarily the same node, gets it.

In reasoning about the performance of our protocols, we associate the cost of zero time with rules that affect only the local state of a node, and some constant time cost with the rules that result in message passing, i.e., the rules that add data to any pair in $O$, the output set.

When considering the performance and responsiveness of a specific system, we restrict the conditions under which certain rules may be applied. These conditions always involve only the local state (and thus can be viewed as specific implementation details). Clearly any resulting behavior of the constrained system is also a possible behavior of the unconstrained system.

We now slightly modify System Message-Passing, replacing rule 3 with rule $3^{\prime}$, Figure 5 , to obtain a responsiveness of $O(N)$ for $N$ nodes.

Lemma 4 System Message-Passing with Rule $3^{\prime}$ has a responsiveness of $O(N)$ for $N$ nodes.

Proof sketch: We assume a cycle graph on $\mathcal{P}$. The claim follows since rule $3^{\prime}$ ensures that $y$ must be the successor of $x$. This yields a responsiveness of $O(N)$. When no node requires the token, it simply cycles around the nodes. As soon as a node does require the token, it will take at most $N$ message delays until the token arrives.

\subsection{Non-deterministic Search}

It is easy to see that the best responsiveness of the system is obtained when the token is always passed to a ready node.
The problem is how does the node with the token know which node requires the token, or, alternatively, how does an active node know who currently has the token.

Rather than have the token cycle over all the nodes, it is possible to send request messages to all the nodes, asking for the token. Each node maintains a set of traps, $W$, indicating which nodes want the token. When a node receives the token, it will forward it to whichever node wants it. The rules for this specification, System Search, are given in Figure 6.

Note that the search messages and traps for now only enable further refinements that will ensure high performance. The non-deterministic nature of the rules permit all kinds of behaviors. We show one optimization (that limits the set of possible behaviors of the system) so that we can get the same responsiveness as in System Message-Passing.

Lemma 5 System Search can be modified to have a responsiveness of $O(N)$ for $N$ nodes.

Proof sketch: To obtain a responsiveness of $O(N)$ for $N$ nodes for System Search, we modify the System with the following restrictions: Rule 4, in which the token can be given to some arbitrary node is disabled (by imposing a precondition false). Rule $3^{\prime}$ from the modified System Message-Passing is added to the system. Rules 5 and 6 are restricted so that they send messages to their cyclic neighbors (by requiring that $y=x^{+1}$ and $u=x^{+1}$ respectively).

The restrictions force the requests to traverse the nodes in a cyclic order. It is now easy to see that within $N$ message delays, the token will be found and send to the requester. 
System Search

\begin{tabular}{|l|ll|}
\hline 0 & Initial state $(Q, P, T, I, O, W)$ & $:\left(\|||\|_{x \in \mathcal{P}}\left(x, \phi_{x}\right),\|\|_{x \in \mathcal{P}}(x, \varnothing), x, \varnothing, \varnothing, \varnothing\right)$ \\
\hline 1 & $\left(Q \mid\left(x, d_{x}\right),-,-,-,-,-\right)$ & $\rightarrow\left(Q \mid\left(x, d_{x} \oplus n e w_{x}\right),-,-,-,-,-\right)$ \\
\hline 2 & $(-,-,-, I, O \mid(x,(y, m),-)$ & $\rightarrow(-,-,-, I \mid(y,(x, m)), O,-)$ \\
\hline 3 & $(-, P|(x,-), \perp, I|(x, y, H)),-,-)$ & $\rightarrow(-, P \mid(x, H), x, I,-,-)$ \\
\hline 4 & $\left(Q\left|\left(x, d_{x}\right), P\right|(x, H), x,-, O,-\right)$ & $\rightarrow\left(Q, P\left|\left(x, H \oplus d_{x}\right), \perp,-, O\right|\left(x,\left(y, H \oplus d_{x}\right)\right),-\right)$ \\
\hline \hline 5 & $\left(Q \mid\left(x, d_{x}\right),-,-,-,-, O, W\right)$ & $\rightarrow\left(-,-,-,-, O\left|\left(x,\left(y, \tau_{x}\right)\right), W\right|\left(x, \tau_{x}\right)\right)$ \\
\hline 6 & $\left(-,-,-, I \mid\left(x,\left(y, \tau_{z}\right)\right), O, W\right)$ & $\rightarrow\left(-,-,-, I, O\left|\left(x,\left(u, \tau_{z}\right)\right), W\right|\left(x, \tau_{z}\right)\right)$ \\
\hline 7 & $\left(-, P|(x, H), x,-, O, W|\left(x, \tau_{y}\right)\right)$ & $\rightarrow(-, P|(x, H), \perp,-, O|(x,(y, H)), W))$ \\
\hline
\end{tabular}

Figure 6: System Search, non-deterministic token search are implemented as Rules 5, 6, and 7. Rule 5: Generate interest by setting trap $\tau_{x}$ for node $x$ and send a message to some other node to trap and return the message. Rule 6: If asked, set a trap locally for the token and continue to ask some other node. Rule 7: If have trap and token then remove trap and send token.

\subsection{Binary Search}

The circular token rotation restriction of System Search obtains linear responsiveness, which is inherent in the sequential search strategy. A responsiveness of $O(\log n)$ is possible by doing a parallel search on the ordered nodes; a node recursively asks two other nodes to search for the token. However, such a search requires an unreasonable number of messages, in the worst case it takes $\Theta(n)$ messages for each node that requires the token and there can be many bottlenecks as well.

It is somewhat surprising that a more efficient protocol results from combining the two modified protocols. That is, the token flows around a ring. Whenever a node wants the token, it sends special "gimme" messages to the node directly across the (logical) ring. A node receiving a special message either returns the token, if it has it, or it lays a local "trap" and sends a "gimme" message halfway around either in the clockwise or counter-clockwise direction depending on whether the token has been at the node before or after it being at the requesting node. The token continues to flow around the ring again from where it was first intercepted. Figure 7 gives the precise rules - we call this specification System BinarySearch (Figure 8 illustrates rule 6 of the system).

There are several desirable properties of our binary search protocol.

\section{Theorem 1 System BinarySearch satisfies the prefix property.}

Proof sketch: Follows by mapping states to the less restricted protocol.

Lemma 6 In System BinarySearch each token request is forwarded $O(\log N)$ times for $N$ nodes.

Proof sketch: If the token does not move, i.e., Rule 4 is not invoked, then within $\log N$ message cycles, a search message will reach the node that has the token. If the token is moving then it will bump into a trap within $\log N$ message cycles.

Theorem 2 System BinarySearch with the additional requirement that the token traps are locally stored and processed in FIFO order, has a responsiveness of $O(\log N)$ for $N$ nodes.

Proof sketch: This follows from the constraint of System BinarySearch and Lemma 6.

Theorem 3 System Binary Search is $\log N$ fair. That is, during the time when some node $x$ wants the token, time $t_{0}$, and gets it, time $t_{1}$, no one node gets the token more than $\log N$ times, and there are no more than $N$ possessions of the token by other nodes.

Proof sketch: We need to show that some node cannot get the token more than $\log N$ times while some other node wants it. Suppose the token is at node $x$, that node $r$ continually requests the token, and that node $w$ also requests the token. Within $\log N$ message steps, the request from node $w$ will arrive at node $x$ by the binary search. Assuming that nodes handle messages and satisfy requests in FIFO order, then when node $r$ returns the token, it will be sent to node $w$.

Similarly, if all nodes search for the token, then node $w$ 's request will be satisfied within $N+\log N$ message steps. $\square$

It is also possible to have nodes keep their requests local and have the token find which node wants it. That is, if a node has the token, it sends out probing messages to see which node wants the token. Once again, if the token or some dummy request cycles around the nodes, then a binary search can be performed requiring only logarithmic number of messages rather than linear. Finally, it is possible to combine both schemes.

\subsection{Simulation results}

We have conducted an empirical study by implementing a simulation of System Binary Search and the regular token 
System BinarySearch

\begin{tabular}{|l|ll|}
\hline 0 & Initial state $(Q, P, T, I, O, W)$ & $:\left(\|\|_{x \in \mathcal{P}}\left(x, \phi_{x}\right),\|\|_{x \in \mathcal{P}}(x, \varnothing), x, \varnothing, \varnothing, \varnothing\right)$ \\
\hline 1 & $\left(Q \mid\left(x, d_{x}\right),-,-,-,-,-\right)$ & $\rightarrow\left(Q \mid\left(x, d_{x} \oplus n e w_{x}\right),-,-,-,-,-\right)$ \\
\hline 2 & $(-,-,-, I, O \mid(x,(y, m)),-)$ & $\rightarrow(-,-,-, I \mid(y,(x, m)), O,-)$ \\
\hline 3 & $(-, P|(x,-), \perp, I|(x,(y, H)),-,-)$ & $\rightarrow(-, P \mid(x, H), x, I,-,-)$ \\
\hline 4 & $\left(Q\left|\left(x, d_{x}\right), P\right|(x, H), x,-, O,-\right)$ & $\rightarrow\left(Q, P\left|\left(x, H \oplus d_{x}\right), \perp,-, O\right|\left(x,\left(x^{+1}, H \oplus d_{x}\right)\right),-\right)$ \\
\hline \hline 5 & $(-, P \mid(x, H),-,-, O, W)$ & $\rightarrow\left(-, P|(x, H),-,-, O|\left(x,\left(y, N, H, \tau_{x}\right)\right), W \mid\left(x, \tau_{x}\right)\right)$ \\
\hline 6 & $\left(-, P|(x, H),-, I|\left(x,\left(y, n, H_{z}, \tau_{z}\right)\right), O, W\right) \rightarrow\left(-, P|(x, H),-, I, O|\left(x,\left(u, \frac{n}{2}, H_{z}, \tau_{z}\right)\right), W \mid\left(x, \tau_{z}\right)\right)$ \\
& & where $u=x^{-n / 2}$ if $H \subset_{C} H_{z}$, \\
& & and $u=x^{+n / 2}$ if $H_{z} \subset_{C} H$ \\
\hline 7 & $\left(-, P|(x, H), x,-, O, W|\left(x, \tau_{y}\right)\right)$ & $\rightarrow(-, P|(x, H), \perp,-, O|(x, \hat{y}, H)), W))$ \\
\hline 8 & $\left(Q\left|\left(x, d_{x}\right), P\right|(x,-), \perp, I \mid(x,(\hat{y}, H)), O,-\right) \rightarrow\left(Q, P\left|\left(x, H \oplus d_{x}\right), \perp, I, O\right|\left(x,\left(y, H \oplus d_{x}\right)\right),-\right)$ \\
\hline
\end{tabular}

Figure 7: System BinarySearch, is a combination of a circular token rotation and a binary search for the token. The first four rules are the same as in System Search. The more complicated search messages contain the local prefix history of the requesting node. Rule 6 compares what the requesting node has seen with what $x$ has seen to decide how to continue the search. ( $A \subset_{C} B$ is the prefix comparison of histories when the histories are projected onto the circular token ring rotation events.) Rule 7 sends the token to the requester indicated by the trap $\tau_{y}$; the decorated $\hat{y}$ indicates that the token is to be returned upon use. Rule 8 gets the token in response to a search request and then immediately returns it to the sender.

(a)

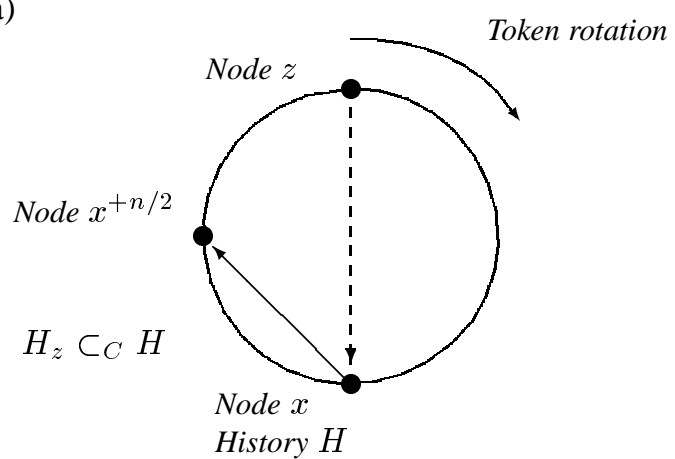

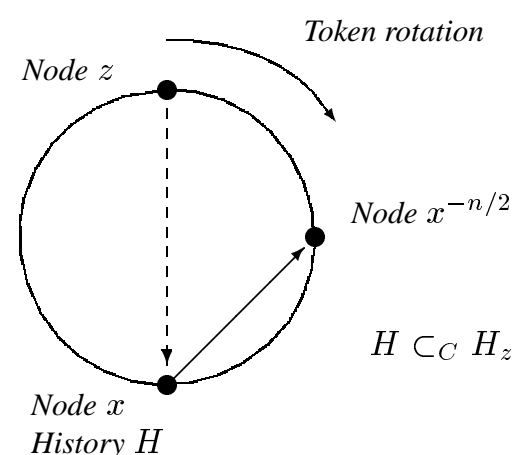

(b)

Figure 8: Illustration of Rule 6 of System BinarySearch: (a) the history $H_{z}$ of the requester at the time of request is a prefix of the history $H$ at node $x$ (when projected onto the token circulation events) - search continues clockwise; (b) the history $H$ at node $x$ is a prefix of the history $H_{z}$ of the requester at the time of request - search continues counter-clockwise.

rotation protocol. Their behavior is observed under various load scenarios, Figures 9 and 10. In each simulation 1000 rounds were performed, i.e., the token visited each node at least 1000 times. Figure 9 presents the results of a simulation where the load is fixed so that on average, every 10 time units, one of the nodes in the system makes a request. The curves show, that using a regular ring algorithm, the average responsiveness approaches 10 , the average distance between nodes on the ring that make a request. Using System Binary Search, the average responsiveness is bounded by $\log n$, where $n$ is the number of processors. This is further illustrated in Figure 10. Here we decrease the load and fix the number of processors $(n=100)$. Using System Binary Search, the average responsiveness approaches $\log n$ from below. For the regular ring algorithm the average responsiveness approaches $n / 2(=50)$.

\subsection{Optimization considerations}

In this section we briefly discuss some of the numerous possible optimization and refinement alternatives for our nondeterministic and binary search protocols. First of all, we have utilized unbounded-size sets and histories for simplicity of presentation. It is easy to bound the size of the sets by throttling the requests and messages issued at the nodes. For the ring protocols the histories can be bounded by introducing the notion of a round and using round counters.

The protocol given by System BinarySearch can be viewed as a delegated search. Here, the search message, once issued by the requesting node, finds its way to the token with the help of other nodes. Nodes remember the search requests they receive from other nodes in the form of traps that are stored locally. To reduce the storage cost of the protocol we want to garbage-collect the obsolete traps. This gives rise to two different algorithms:

Token-rotation clean up. Here the token itself is used to remove old traps during subsequent token rotation 


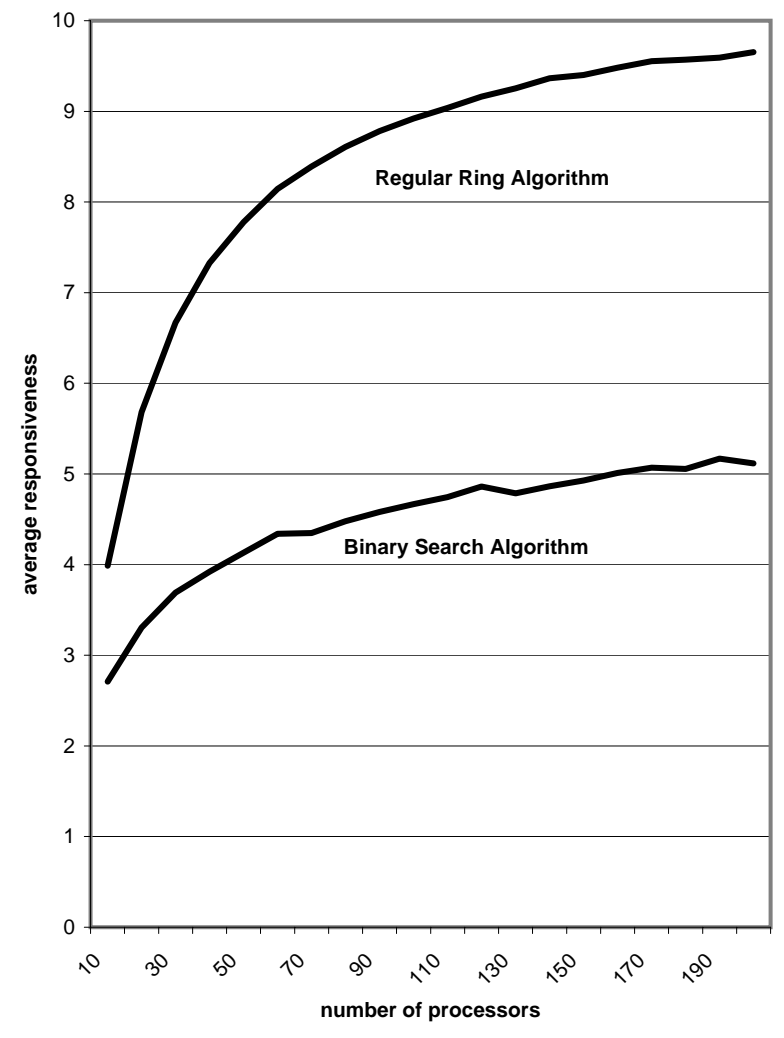

Figure 9: Performance with fixed load

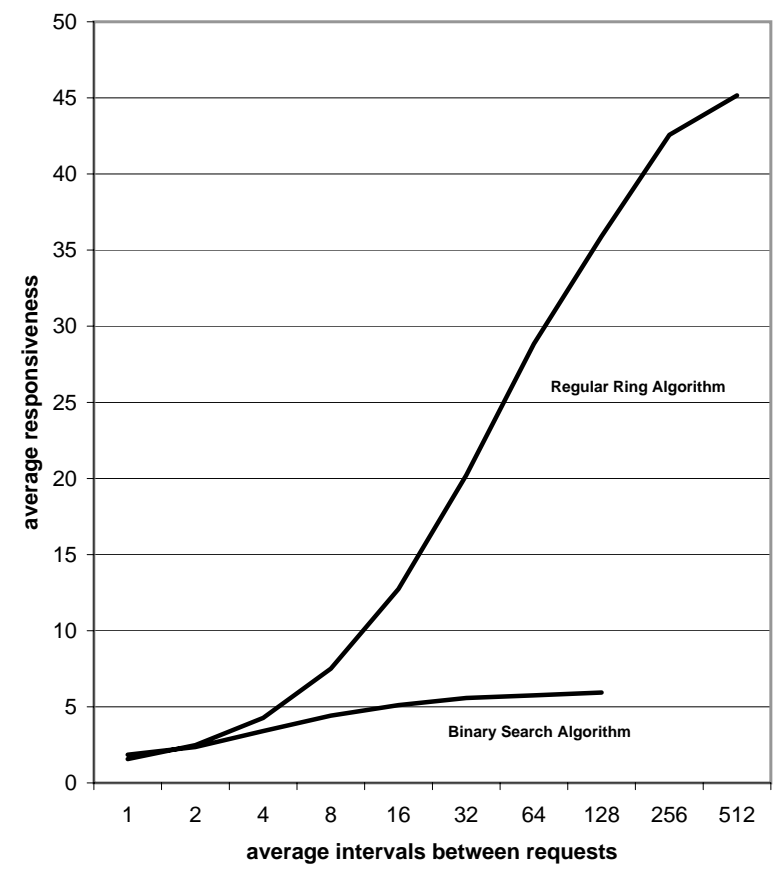

Figure 10: Performance with fixed number of processors rounds. The token can carry enough information, e.g., round number, information about the satisfaction of a search request, to enable the nodes to clean up.

Inverse token clean up. The token after, it was found, is not passed directly to the requesting node, but rather traces the trail left behind by the search messages. In doing so, it removes the traps en route to the requester.

Another modification to the protocol would be the directed search, where search messages do not migrate through the ring but instead are always returned to the searching node informing it whether the token was found or not. This increases (doubles) the number of search messages from $\log N$ to $2 \log N$ in the worst case, however if the token reaches the searching node during the search due to its normal rotation, the search can be stopped, thus saving some number of messages.

In yet another further extension, nodes ensure that they have only one request (one "gimme" message) outstanding. All other requests that arrive are delayed until the first request has been satisfied. This reduces the number of "gimme" messages to be no more than the number of token passing messages.

Finally, we note that the speed of token passing around the cycle can be varied according to the demand - very slow when only a few nodes require the token and much faster when there is high demand.

\section{Conclusion}

The methodology followed in this paper provides wide scope for performance optimizations and adaptive tuning. In particular, adaptive algorithms can often be immediately derived from the abstract specifications in a methodical way that preserves correctness. The methodology in this paper is based on the refinements to specifications stated as transition systems. In this paper we use the Term Rewriting Systems (TRS) [2] framework to formally express specifications. We note that we could have done the same, maybe not as succinctly as presented here, using Temporal Logic of Actions (TLA) [11], or Input/Output Automata (IOA) $[12,14]$.

We have specified a family of token passing protocols. One interesting observation is that messages are used in two different ways. One is to pass the token (potentially with the message history). Another is to push the system along certain performance optimized trajectories. The first requires that messages arrive correctly, or at least with a high probability as well as a mechanism to resend in case of failure (not considered in this paper). As is usually the case, raising the likelihood of success is more expensive in terms of several different resources. The second can be accomplished using "lightweight" messages, since they do not impact system correctness, only its performance.

Token-passing protocols, as sometimes considered in the literature, trade token access efficiency for loads at certain nodes, e.g., as is the case with fixed tree-based topologies where fast access comes at the cost of high loads at 
the roots. Our token-passing scheme is able to capture in a single protocol the good load balancing and fairness property of the ring, with the efficiency of logarithmic access. This is accomplished in a fluid way, where the tokenpossessing nodes can temporarily act as virtual roots of a token-distribution tree.

Our future plans include making the protocols more dynamic with respect to the nodes comprising the network. It is possible to modify the protocol to handle nodes that asynchronously leave and join the group. The search mechanism needs to know those nodes that are halfway, $1 / 4$ way, etc., around the cycle. An approximation may be sufficient, and so nodes need only a set of a logarithmic number of neighbors. Note also that by combining token traversal with searching, the protocol already has a way of handling failures. If a node $x$ with the token fails, then nothing will happen until some other node $y$ needs the token, at which point it will quickly discover that the token holder has failed (provided a time-out based detection is available). Node $y$ will then get in touch with $x^{-1}$ and $x^{+1}$. These two nodes can then determine if $x$ is really dead and if the token was at $x$. If so, they can generate a new token.

Acknowledgments: We thank Greg Malewicz for several valuable observations and comments.

\section{References}

[1] Y. Amir, L. E. Moser, P. M. Melliar-Smith, D. A. Agarwal and P. Ciarfella, "The Totem single-ring ordering and membership protocol," ACM Transactions on Computer Systems, vol. 13, no. 4, November 1995, pp. 311-342.

[2] Arvind and X. Shen, "Design and Verification of Processors Using Term Rewriting Systems", CSG Memo 419, LCS, MIT; to appear in IEEE Micro, 1999.

[3] S. Banerjee and P. K. Chrysanthis, "A New Token Passing Distributed Mutual Exclusion Algorithm ”, In Proc. of the 16th International Conference on Distributed Computing Systems, pp. 717-724, Hong Kong, May 27-30, 1996.

[4] H. Buhram, M. Franklin, J.A. Garay, J.-H. Hoepman, J. Tromp and P. Vitanyi, "Mutual Search", J. ACM, vol. 46, no. 4, pp. 517-536, 1999.

[5] Y-I. Chang, M. Singhal, and M. T. Liu. "A dynamic tokenbased distributed mutual exclusion algorithm", In Proc. 10th IEEE Int'l Phoenix Conf. on Computers and Communications, pp. 240-246, 1991.

[6] Communications of the ACM, special section on group communications, vol. 39, no. 4, 1996.

[7] F. Commoner, A.W. Holt, S. Even and A. Pnueli, "Marked directed graphs", JCSS, vol. 5, no. 6, pp. 511-523, 1971.

[8] J.-M. Helary and A. Mostefaoui, "A $O\left(\log _{2} n\right)$ fault-tolerant distributed mutual exclusion algorithm based on open-cube structure", Research Report 2041, INRIA, September 1993.

[9] J.-M. Helary, A. Mostefaoui and M. Raynal, "A General Scheme for Token- and Tree-based Distributed Mutual Exclusion Algorithms," Research Report 1692, INRIA-IRISA, May 1992. Also in IEEE Transactions on Parallel and Distributed Systems, Vol. 5, No. 11, pp. 1185-1196, 1994.

[10] L. Lamport, "Time, clocks, and the ordering of events in a distributed system", Comm. ACM, vol. 21, no. 7, pp. 558$565,1978$.
[11] L. Lamport, "The Temporal Logic of Actions", ACM Transactions on Programming Languages and Systems, 16(3):872-923, 1994.

[12] N.A. Lynch, Distributed Algorithms, Morgan Kaufman Publishers, San Mateo, CA, 1996.

[13] N.A. Lynch and M.R. Tuttle, "Hierarchical Correctness Proofs for Distributed Algorithms", in Proc of the 6th Annual ACM Symp. on Principles of Distributed Computing, pp. 137-151, 1987.

[14] N.A. Lynch and M.R. Tuttle, "An Introduction to Input/Output Automata", CWI Quarterly, vol.2, no. 3, pp. 219246, 1989.

[15] G. Peterson and M. Fischer, "Economical Solutions for the Critical Section Problem in a Distributed System", in Proc of the 9th Annual ACM Symp. on Theory of Computing, pp. 91-97, 1977.

[16] M. Raynal, Algorithms for Mutual Exclusion, MIT Press, 1986.

[17] X. Shen, Arvind and L. Rudolph "CACHET: An Adaptive Cache Coherence Protocol for Distributed Shared-Memory Systems", In proceedings of the 13th ACM SIGARCH International Conference on Supercomputing, June 1999, Rhodes, Greece, 1999.

[18] M. Singhal, "A Taxonomy of Distributed Mutual Exclusion", Journal of Parallel and Distributed Computing 18(1), pp. 94101, 1993.

[19] R. Vitenberg, I. Keidar, G.V. Chockler and D. Dolev, "Group Communication Specifications: A Comprehensive Study", MIT Technical Report MIT-LCS-TR-790, September 1999. URL http://theory.lcs.mit.edu/ idish/ftp/gcs-survey-tr.ps. 